\title{
Editorial
}

\section{The diagnosis of cirrhosis: clinical relevance and methodology}

\author{
Solko W. Schalm \\ Department of Hepatogastroenterology and Internal Medicine, Erasmus University Hospital -- Dijkzigt, Rotterdam, The Netherlands
}

\section{$\mathrm{C}$} IRRHOSIS is defined as a diffuse process characterized by fibrosis and alteration of normal liver architecture into structurally abnormal nodules of liver cells surrounded by fibrosis (1). The diagnosis of cirrhosis by liver biopsy is made if the biopsy contains at least one nodule completely surrounded by fibrosis tissue; large fibrous septae with absence of portal tracts, altered hepatic architecture and loss of vascular relationships arc features suggestive of, but not diagnostic for cirrhosis (1).

Cirrhosis is usually divided into compensated and decompensated cirrhosis. In the latter stage, patients are symptomatic, with jaundice, ascites, variceal bleeding, or encephalopathy; the clinical significance of this stage needs little discussion in view of its high 1-year mortality. Consideration of liver transplantation is desirable in this group, especially if the etiology of the liver disease indicates that it is not amenable to medical therapy.

The clinical significance of compensated cirrhosis is less well recognized, as more than $70 \%$ of patients are asymptomatic (2). Such patients often have diseases such as chronic hepatitis $\mathrm{B}$, chronic hepatitis $\mathrm{C}$ or autoimmune hepatitis.

In the nineties, it has become increasingly apparent that the stage of cirrhosis is a key factor for defining prognosis and management; this is similar to the situation in the seventies when the etiology of the liver disease emerged as a clinically important factor for prognosis and response to therapy (3). With regard to prognosis, mortality in long-term follow-up studies of 'chronic active hepatitis' is confined mainly to patients with cirrhosis at entry (4). A recent study of a cohort of more than 200 patients with primary intrahepatic

Correspondence: Prof. dr. S. W. Schalm, Department of Hepatogastroenterology and Internal Medicine, University Hospital Rotterdam - Dijkzigt, P.O. Box 2040, 3000 CA Rotterdam, The Netherlands. Tel: $31 \quad 104635942$. Fax: 3110436 5916. e-mail: schalm@inw2.azr.nl cholangitis (PBC) treated with ursodeoxycholic acid for 5 years confirmed the finding that mortality and clinical complications of liver disease occurred predominantly in those with cirrhosis (5).

In relation to response to therapy, two recent consensus reports on the management of chronic hepatitis $\mathrm{C}$ recommended alfa interferon therapy for patients with active disease and signs of progression (fibrosis), but not for patients with cirrhosis $(6,7)$. The biological basis for the recommendation is the significantly lower response to alfa interferon in cirrhosis.

Concerning monitoring, the diagnosis of cirrhosis in a patient with chronic liver disease is nowadays of clinical significance for those patients who elect to participate in screening programs for detection of hepatocellular carcinoma (8).

In view of the increasing clinical importance of the diagnosis of cirrhosis in the asymptomatic patient, it is disheartening to realize that diagnostic accuracy in routine clinical practice is rather poor. While we require a sensitivity of more than $95 \%$ for etiologic tests like HBsAg and anti-HCV, we commonly use methodology (percutaneous liver biopsy) with a sensitivity of below $80 \%$ in detecting cirrhosis. The diagnostic sensitivity can be increased by examining more than one biopsy specimen $(9,10)$ or by combining liver biopsy with laparoscopy (11), but neither approach has found general acceptance in routine clinical practice.

The paper by Gaiani et al. (12) in this issue of the Journal may provide a solution to the clinical dilemma of diagnosing cirrhosis. They performed abdominal ultrasound prior to taking a percutanous liver biopsy in 212 patients with chronic liver disease without clinical signs suggestive of cirrhosis. The diagnosis of cirrhosis was made in 47 patients by histology, and in 69 patients by ultrasound. The histological criteria for cirrhosis were based on the international standard; the ultrasound criteria for cirrhosis were initially based on a score to which 7 parameters contributed. After completion of the study, it was found that only two ultra- 
sound variables, liver surface nodularity and portal vein mean flow velocity, made independent contributions to the diagnosis.

In 37 patients, histology and ultrasound were concordant in the diagnosis of cirrhosis. In 10 patients cirrhosis was found on histology, but ultrasound showed no conclusive evidence (false-negative ultrasound). In 32 patients, ultrasound indicated cirrhosis, but histology was not diagnostic. The authors correctly stated that it is not clear whether ultrasound overestimates the diagnosis of cirrhosis, or histology underestimates it. However, there is convincing evidence that overestimation of the diagnosis of cirrhosis by ultrasound is unlikely:

- The key element in the ultrasound diagnosis is the nodularity of the liver surface;

- Clinical and biochemical signs of liver cirrhosis developed with equal frequency during 6 months of follow-up in patients with histologic cirrhosis, as in patients with cirrhosis based on ultrasound only;

- In 29 out of 32 patients with cirrhosis based on ultrasound only, the histology showed sample fragmentation, advanced fibrosis, and architectural distinction, but no well-defined nodules.

Thus, assuming that at least 29 patients with a diagnosis of cirrhosis by ultrasound did in fact have cirrhosis, the real number of patients with cirrhosis in the cohort was at least 76 . So, the prevalence of cirrhosis in the cohort was $36 \%(76 / 212)$, instead of $22 \%(47 /$ 212). Standard calculations then revealed a diagnostic sensitivity of $62 \%$ for percutaneous liver biopsy, and $87 \%$ for ultrasound; specificities for both methods are $98 \%$ or more.

The gold standard for the diagnosis of cirrhosis in hepatology is the liver biopsy. In view of these findings, do we need to adapt? The design of Gaiani's study was excellent, with a training and a validation sample. The statistical analysis evaluated the contribution of each element of the ultrasound score, and defined the two independent variables with key prognostic information. The study confirms the underestimation of the diagnosis of cirrhosis by a single specimen. Therefore, in view of the clinical importance of making the diagnosis of cirrhosis, we need to adapt our procedures.

What should become the routine clinical practice? Prior to performing a liver biopsy, ultrasound should be done by an operator trained to assess liver surface nodularity and possibly portal vein mean flow velocity. The result should be available at the time of liver biopsy. If cirrhosis is suspected from the ultrasound or the clinical evaluation, at least two separate specimens should be obtained for standard histological elevation. If histology shows no cirrhosis (nodules surrounded by fibrosis), but shows fragmentation, fibrosis, and architectural distortion, the diagnosis of cirrhosis should still be made if there is ultrasound diagnosis of cirrhosis. It is to be hoped that this approach will eliminate histological diagnosis as early, beginning, possible and probable cirrhosis. Hepatologists now have a refined set of tests to unravel the etiology of liver disease; the time has come to improve our methods for determining the stage of the liver disease, specifically the diagnosis of cirrhosis.

\section{References}

1. Diseases of the Liver and Biliary Tract. Standardization of Nomenclature, Diagnostic Criteria and Prognosis. New York: Raven Press; 1994.

2. Realdi G, Fattovich G, Hadziyannis S, Schalm SW, Almasio $\mathbf{P}$, Sanchez-Tapias $\mathrm{JM}$, et al. and the Investigators of the European Concerted Action on Viral Hepatitis (Eurohep). Survival and prognostic factors in 366 patients with compensated cirrhosis type B: a multicenter study. J Hepatol 1994; 21: 656-66.

3. Schalm SW, Summerskill WHJ, Gitnick GL, Elveback LR. Contrasting features and responses to treatment of chronic active liver disease with and without hepatitis Bs antigen. Gut 1976; 17: 781-6.

4. Keating JJ, O'Brien CJ, Stellon AJ, Portmann BC, Johnson $\mathrm{RD}$, Johnson PJ, et al. Influence of aetiology, clinical and histological features on survival in chronic active hepatitis: an analysis of 204 patients. Q J Med 1987; 237: 59-66.

5. Hoogstraten HJF van, Buuren $H R$ van, Kate FJW ten, Hansen BE, Berge Henegouwen GP van, Schalm SW. Fiveyear sustained biochemical improvement in ursodeoxycholic acid (UDCA)-treated primary biliary cirrhosis (PBC) does not preclude disease progression. J Hepatol 1997; 26 (Suppl 1): 169 .

6. Hépatite C: dépistage et trâitement. Gastroenterol Clin Biol 1997; 20: S202-211.

7. Management of Hepatitis C. National Institutes of Health Consensus Development Conference Statement, 1997. http.// consensus.nih.gov.

8. Zoli M, Magalotti D, Gueli C, Marchesini G, Pisi E. Efficacy of a surveilance program for early detection of hepatocellular carcinoma. Cancer 1996; 78: 977-85.

9. Soloway RD, Baggenstoss AH, Schoenfield LJ, Summerskill WHJ. Observer error and sampling variability tested in evaluation of hepatitis and cirrhosis by liver biopsy. Dig Dis Sci 1971; 16: 1082-6.

10. Maharaj B, Maharaj RJ, Leary WP, Cooppan RM, Naran $\mathrm{AD}$, Pirie D, et al. Sampling variability and its influence on the diagnostic yield of percutaneous needle biopsy of the liver. Lancet 1986; i: 523-5.

11. Pagliaro L, Rinaldi F, Craxi A, Di Piazza S, Filippazzo G, Gatto $G$, et al. Percutaneous blind biopsy versus laparoscopy with guided biopsy in diagnosis of cirrhosis. A prospective, randomized trial. Dig Dis Sci 1983; 28: 39-43.

12. Gaiani S, Gramantieri L, Venturoli N, Piscaglia F, Siringo S, D'Errico A, et al. What is the criterion for differentiating chronic hepatitis from compensated cirrhosis? A prospective study comparing ultrasonography and percutaneous liver biopsy. J Hepatol 1997; 6: 979-985. 